\section{Akademisierung in der Pflege sehen Ärzte kritisch}

\section{Besitzstandswahrung, Angst vor Konkurrenz und Machtverlust - Ärzte sehen die Akademisierung der Pflegeberufe noch immer mehr als Gefahr denn als Chance. Darauf deutet eine Auswertung des „Deutschen Ärzteblatts“.}

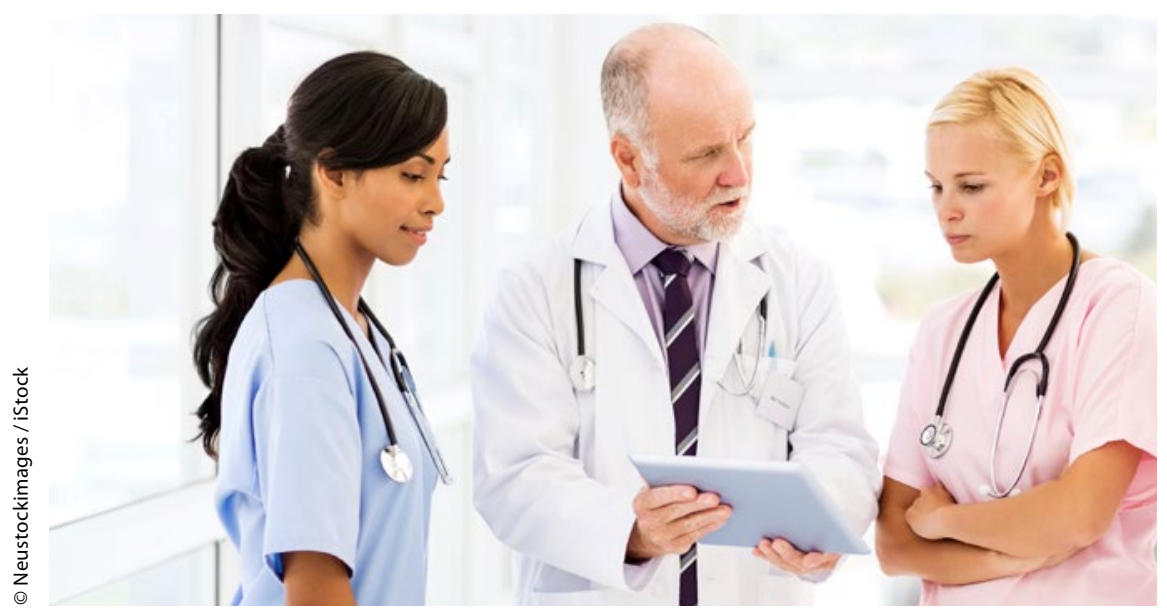

Starre Hierarchien, ein autokratischer Führungsstil und zum Teil die althergebrachten Rollenbilder zwischen Mann und Frau scheinen im deutschen Gesundheitswesen überdauert zu haben.

A $\mathrm{n}$ einer besseren Qualifikation von Pflegenden haben auch Ärzte nichts auszusetzen - solange es um die Arbeit am Krankenbett geht. Aber das Rollenverständnis soll so bleiben, wie es ist: Der Arzt sagt auch weiterhin, wo es lang geht, von einer Zusammenarbeit auf Augenhöhe wollen die meisten offenbar nichts wissen. Das lässt sich aus einer Analyse von 30 Artikeln schließen, die zur Akademisierung und Professionalisierung der Pflege in den vergangenen Jahren im „Deutschen Ärzteblatt" erschienen sind.

Zwar würden demografische und gesundheitsökonomische Gründe, die woanders längst zu einer höheren Qualifizierung der patientennahen Pflege geführt hätten, auch auf das deutsche Gesundheitswesen zutreffen, schreiben Professor Anke Simon und Bettina Flaiz von der Dualen Hochschule BadenWürttemberg, Stuttgart, in ihrer Analyse. Jedoch scheinen ihrer Ansicht nach „starre Hierarchien, ein autokratischer Führungsstil und zum Teil die althergebrachten Rollenbilder zwischen Mann und Frau im deutschen Gesundheitswesen überdauert zu haben“.

\section{Ärzte fürchten Konkurrenz}

Simon und Flaiz haben sich das „Deutsche Ärzteblatt" vorgenommen, um zu schauen, wie die Professionalisierung der Pflege von Ärzten reflektiert wird. Relativ wenig - so das Ergebnis.

Anfang der 1990er-Jahre findet die Etablierung neuer Pflegestudiengänge ein erstes Echo. So wird beispielsweise darüber diskutiert, pflegerische Lehrund Leitungskräfte per Hochschulabschluss zu qualifizieren. Die Bundesärztekammer (BÄK) wird als Unterstützerin des Vorhabens im „Ärzteblatt" genannt. „Sie widerspricht jedoch Forderungen, die Krankenpflegeberufe generell in den Stand von Akademikern zu versetzen", heißt es in einem Artikel. Darin wird auch der Präsident der Deutschen Zentrale für Volksgesundheit (DZV) zitiert. Er hat Angst, dass die besser qualifizierten Pflegenden ihre „ureigenen Tätigkeiten und ihren Einsatz am Krankenbett vernachlässigen und danach trachten, arztgleiche oder arztähnliche Verrichtungen $\mathrm{zu}$ übernehmen (mit entsprechenden Bezahlungsforderungen)“.
Diese Argumentation setzt sich dann über die Jahre fort. Zudem befürchtet etwa der langjährige Marburger-BundVorsitzende Frank Ulrich Montgomery, dass es zu einem Hierarchiegerangel kommt, wenn akademische und nichtakademische Pflegende nebeneinanderstehen. „Wir brauchen nicht nur Hochqualifizierte, wir brauchen auch Menschen die pflegen", wird Montgomery in einem anderen Artikel zitiert.

Nach 2005 beginnt eine neue Diskussion um die Aufgabenverteilung. In einem Beitrag stellt sich die Landesärztekammer Rheinland-Pfalz gegen ein Modellprojekt, in dem Pflegekräfte die Anamnese erheben. „Es drohen der Ausverkauf ureigener ärztlicher Leistungen und ernsthafte Qualitätseinbußen.“

\section{Besorgniserregende Innovationsbremse}

Es gibt aber auch Ärzte, die einer weiteren Professionalisierung offener gegenüberstehen. So fordert der Wissenschaftsrat im Jahr 2012 eine Akademikerquote von $10-12 \%$ im Pflegebereich. Der Neurologe Professor Hans-Jochen Heinze, damals Vorsitzender einer entsprechenden Sachverständigenkommission, verteidigt diese Haltung: „Es geht uns um die Qualifikation am Bett. Da ist noch eine Menge Potenzial vorhanden, das bei uns einfach nicht ausgeschöpft wird." Diese Aussage stößt wiederum Ärzteblatt-Lesern und der Bundesärztekammer auf: „Es ist sachlich nicht begründbar, warum ein weltweit anerkanntes und funktionierendes dreijähriges duales Ausbildungssystem durch ein dreijähriges akademisches Studium ersetzt werden soll." Ein Leser vermutet sogar, es gehe der Pflege ausschließlich um die Umgestaltung der Machtverhältnisse im Gesundheitswesen.

Das Fazit von Simon und Flaiz nach der Lektüre von rund 25 Jahren „Ärzteblatt“: Die Ärzteschaft hält nach wie vor an traditionellen Rollenbildern fest, und dies stelle eine „zunehmend besorgniserregende Innovationsbremse" dar.

Anke Simon, Bettina Flaiz. Sichtweisen der Ärzteschaft zur Professionalisierung der Pflege. HeilberufeSCIENCE (2015) 6:86-93. DOI 10.1007/ s16024-015-0253-4 